\title{
THE TANGENT CONE OF A LOCAL RING OF CODIMENSION 2
}

\author{
MOUSUMI MANDAL AND MARIA EVELINA ROSSI
}

Dedicated to Professor Ngo Viet Trung on the occasion of his 60th birthday

\begin{abstract}
Let $(S, \mathfrak{n})$ be a regular local ring and let $I \subseteq \mathfrak{n}^{2}$ be a perfect ideal of $S$. Sharp upper bounds on the minimal number of generators of $I$ are known in terms of the Hilbert function of $R=S / I$. Starting from information on the ideal $I$, for instance the minimal number of generators, a difficult task is to determine good bounds on the minimal number of generators of the leading ideal $I^{*}$ which defines the tangent cone of $R$ or to give information on its graded structure. Motivated by papers of S.C. Kothari, S. Goto et al. concerning the leading ideal of a complete intersection $I=(f, g)$ in a regular local ring, we present results provided ht $(I)=2$. If $I$ is a complete intersection, we prove that the Hilbert function of $R$ determines the graded Betti numbers of the leading ideal and, as a consequence, we recover most of the results of the previously quoted papers. The description is more complicated if $\nu(I)>2$ and a careful investigation can be provided when $\nu(I)=3$. Several examples illustrating our results are given.
\end{abstract}

\section{INTRODUCTION AND NOTATION}

Let $(R, \mathfrak{m}, k)$ be a local ring with maximal ideal $\mathfrak{m}$ and residue field $k$. The associated graded ring $G=\operatorname{gr}_{\mathfrak{m}}(R)=\oplus_{i \geq 0} \mathfrak{m}^{i} / \mathfrak{m}^{i+1}$ corresponds to a relevant geometric construction in the case $R$ is the localization at the origin $\mathrm{O}$ of the coordinate ring of an affine variety $V$ passing through $\mathrm{O}$. It turns out that $G$ is the coordinate ring of the Tangent Cone of $V$ at $\mathrm{O}$, which is the cone composed of all lines that are the limiting positions of secant lines to $V$ in $O$. Consider a minimal Cohen presentation $S / I$ of $R$ where $(S, \mathfrak{n}, k)$ is a regular local ring and $I \subseteq \mathfrak{n}^{2}$ is an ideal of $S$. We recall that $G \simeq P / I^{*}$ where $P=\operatorname{gr}_{\mathfrak{n}}(S)$ is the polynomial ring and $I^{*}$ is the homogeneous ideal of $P$ generated by the initial forms of the elements of $I$. From the algebraic point of view, the local ring $R$ and the standard graded $k$-algebra $G$ share the same Hilbert function. In fact, by

2010 Mathematics Subject Classification. 13A30,13D02, 13H10.

Key words and phrases. Minimal free resolution, Associated graded ring, Minimal number of generators, Hilbert function.

The first author was supported by INdAM-COFUND Marie-Curie Fellowship. The second author was supported by MIUR, PRIN 2010-11 (GVA). This work was partly accomplished while the first author was visiting the University of Genoa. 
definition, the Hilbert function of $R$ is the numerical function $H F: \mathbb{N} \rightarrow \mathbb{N}$ such that $H F_{R}(i)=\operatorname{dim}_{k} \mathfrak{m}^{i} / \mathfrak{m}^{i+1}$.

We address our interest to the structure of $I^{*}$, called the leading ideal of $I$, which encodes the algebraic and the geometric information on $I$. Denote by $\nu(I)$ the minimal number of generators of $I$, then by a classical result of Krull and by the definition of $I^{*}$, it is known that

$$
\operatorname{ht}(I) \leq \nu(I) \leq \nu\left(I^{*}\right) .
$$

Upper bounds for $\nu\left(I^{*}\right)$ (and hence for $\nu(I)$ ) are known in terms of the multiplicity of $R$ or, more precisely, in terms of the Hilbert function of $R$, see for instance [2], [6], [7], [15], [16].

Starting from information on the ideal $I$, a more difficult task is to prove sharp lower bounds for $I^{*}$ improving $\nu(I) \leq \nu\left(I^{*}\right)$, since this involves the study of the structure of $I^{*}$.

The work of Goto et al. (see [8], [9], 10]) evidences the difficulty of the problem, even if we assume that $I=(f, g)$ is generated by a regular sequence in a 2-dimensional regular local ring $S$.

More in general, if not otherwise specified, we assume $I \subseteq \mathfrak{n}^{2}$ is an ideal of height 2 (not necessarily a complete intersection) in a $r$-dimensional regular local ring $(S, \mathfrak{n})$ such that $G$ is Cohen-Macaulay. Under our assumption $I$ and $I^{*}$ are homogeneous perfect ideals of codimension two, hence good information come from the Hilbert-Burch theorem. The main results of the paper are contained in Corollary 1.2, Theorem 2.1, Theorem 2.4 and Theorem 3.1 .

Let $R=S / I$ and denote by $H S_{R}(t)=\sum_{j \geq 0} H F_{R}(j) t^{j}$ the Hilbert series of $R$. It is well known that

$$
H S_{R}(t)=\frac{h_{R}(t)}{(1-t)^{r-2}}
$$

where $h_{R}(t)=1+h_{1} t+\cdots+h_{s} t^{s} \in \mathbb{Z}[t]$ is called $h$-polynomial and $h_{R}=\left(1, h_{1}, \ldots, h_{s}\right)$ is called $h$-vector. If $G$ is Cohen-Macaulay of codimension two, then $h_{R}(t)$ represents the Hilbert series of any Artinian reduction of $R$ (we may assume that $k$ is infinite), hence, by Macaulay's inequalities, the $h$-vector

$$
h_{R}=\left(1,2, \ldots, d, h_{d}, \ldots, h_{s}\right) \text { verifies } d \geq h_{d} \geq h_{d+1} \geq \cdots \geq h_{s} \geq 1
$$

where $d$ is the order of $I$, that is the maximum integer such that $I \subseteq \mathfrak{n}^{d}$.

From now on, a numerical function $h$ verifying (2) is called $O$-sequence. Motivated by the above observation, we assume $R$ is an Artinian local ring of embedding dimension two. 
Macaulay proved that, given an $O$-sequence $h$, then there exists an unique lex-segment ideal $L$ in $P$ such that $h=H F_{P / L}$. Then using this fact and the Hilbert-Burch Theorem, it is known that (11) specializes to

$$
\nu(I) \leq \nu\left(I^{*}\right) \leq \nu(L)=d+1 .
$$

The Hilbert function of an Artinian local ring $R$ of embedding dimension 2 has been studied by several authors.

Iarrobino in [11] proved that if $p:=\max \left\{\left|H F_{R}(i)-H F_{R}(i+1)\right| \mid i=1, \ldots, s\right\}$, then

$$
\nu(I) \geq p+1 .
$$

If $h$ is an $O$-sequence and $m$ is any integer verifying $p+1 \leq m \leq d+1$, then Bertella in [1] gave an effective method for constructing an ideal $I \subseteq S=k[|x, y|]$ such that $\nu(I)=m$ and $H F_{S / I}=h$ (see also [14, Remark 4.7]).

In general $\nu\left(I^{*}\right) \geq m=\nu(I)$ and Theorem 1.1 gives a more precise bound. Notice that the ideals $I$ and $I^{*}$ share the same Hilbert function, but in general a different minimal number of generators occurs. For instance, Example 1.4 shows that there exist ideals $I$ whose Hilbert function forces $\nu\left(I^{*}\right) \geq \nu(I)+3$.

As we will explain later, an important tool in our approach is the technique of the consecutive cancellations described by Peeva in [13] in the graded case and, by Rossi and Sharifan, in [14] in the local case. It is known that in codimension two each cancellation can be realized (see [1]).

Notice that if $m=2$, hence $I=(f, g)$ is generated by a regular sequence, we prove that the Hilbert function fixes the graded Betti numbers of $I^{*}$ (see Theorem 2.1). In particular we prove that there is a one to one correspondence between the $O$-sequences $h$ such that $h=H F_{S / I}$ with $I=(f, g)$ and two sequences of integers $\left(c_{1}, \ldots, c_{n}\right)$ and $\left(e_{1}, \ldots, e_{n}\right)$ related by suitable conditions described in Theorem 2.2. The two sequences $\left(c_{1}, \ldots, c_{n}\right)$ and $\left(e_{1}, \ldots, e_{n}\right)$ give also necessary conditions for constructing a complete intersection whose leading ideal has a Betti table determined by these invariants, see Theorem 2.4.

The results recover most of the results proved by S. Goto, W. Heinzer and M.K. Kim in [9] and [10] where many technical computations were necessary. In the quoted papers the authors sharpened a result by Kothari in [12] who answered a question raised by Abhyankar concerning the Hilbert function of the localization of a pair of plain curves. The new approach allows us easier proofs because it takes advantage of the numerical invariants coming from a minimal free resolution of $I^{*}$. 
If $\nu(I)>2$, then the Hilbert function of $R$ does no longer fix the Betti numbers of $I^{*}$ and a careful investigation is done if $\nu(I)=3$.

\section{LOWER BOUND FOR $\nu\left(I^{*}\right)$}

Given a numerical function $h: \mathbb{N} \rightarrow \mathbb{N}$, let us denote by $\triangle h(j)=h(j)-h(j-1)$ the first difference and by $\triangle^{2} h(j)=\triangle h(j)-\triangle h(j-1)=h(j)-2 h(j-1)+h(j-2)$ the second difference operator. Denote by $P$ the polynomial ring $k\left[x_{1}, \ldots, x_{n}\right]$ in $n$ indeterminates. Since $I^{*}$ is a homogeneous perfect ideal of codimension two in the polynomial $\operatorname{ring} P$, for sake of completeness we insert here some general facts that will be useful in the present work.

Let $J$ be a perfect homogeneous ideal of height two in $P$ with minimal $P$-free graded resolution:

$$
\text { F. : } 0 \rightarrow \oplus_{j \geq 0} P^{\beta_{1 j}}(-j) \stackrel{\phi}{\rightarrow} \oplus_{j \geq 0} P^{\beta_{0 j}}(-j) \rightarrow J \rightarrow 0 .
$$

By the Hilbert-Burch Theorem, $J$ can be generated by the maximal minors of an homogeneous matrix associated to $\phi$ of size $m \times(m-1)$ where $m=\sum_{j} \beta_{0 j}$.

Accordingly with [14], we recall that F. admits a negative cancellation (resp. zero cancellation ) if there exist integers $j<j^{\prime}$ (resp. $j=j^{\prime}$ ) such that $\beta_{1 j}, \beta_{0 j^{\prime}}>0$. We will denote it by $\left(P(-j), P\left(-j^{\prime}\right)\right)$.

For example $0 \rightarrow P(-3) \oplus P(-5) \oplus P(-6) \rightarrow P^{2}(-2) \oplus P^{2}(-5) \rightarrow \ldots$ admits a zero cancellation $(P(-5), P(-5))$ and a negative cancellation $(P(-3), P(-5))$.

The following result gives a lower bound for the number of generators of a homogeneous perfect ideal $J$ of codimension two in the polynomial ring $P$ in terms of the second difference operator of its $h$-vector. In particular we prove that when $J$ is minimally generated with respect a bound given by its Hilbert function, then the graded Betti numbers of $J$ are uniquely determined.

We denote by $|n|$ the positive value of the integer $n$.

Theorem 1.1. Let $h:=\left(1,2, \ldots, d, h_{d}, \ldots, h_{s}\right)$ be an $O$-sequence and define the sets $\mathcal{I}:=\left\{j \mid \triangle^{2} h(j) \leq-1\right\}$ and $\mathcal{J}:=\left\{j \mid \triangle^{2} h(j) \geq 1\right\}$. Let $J$ be a perfect homogeneous ideal of height two of $P$ and such that $h$ is the h-vector of $G=P / J$. Then

(1) $\nu(J) \geq \sum_{i \in \mathcal{I}}\left|\triangle^{2} h(i)\right|$

(2) If the equality holds, then the Betti numbers of $J$ are uniquely determined by the Hilbert function and

$$
\beta_{0 j}(J)= \begin{cases}\left|\triangle^{2} h(j)\right| & \text { for } j \in \mathcal{I} \\ 0 & \text { otherwise }\end{cases}
$$




$$
\beta_{1 j}(J)= \begin{cases}\left|\triangle^{2} h(j)\right| & \text { for } j \in \mathcal{J} \\ 0 & \text { otherwise. }\end{cases}
$$

Proof. We may assume that the residue field $k$ is infinite. Since $G$ is Cohen-Macaulay of dimension $n-2$, let $Q=\left(\ell_{1}, \ldots, \ell_{n-2}\right)$ be an ideal of $P$ generated by linear forms such that $\bar{Q}=Q+J / J$ is generated by a maximal $G$-regular sequence. Denote $\bar{G}=G / \bar{Q}$ and $\bar{J}=J+Q / Q$. Now the following facts hold:

- $\beta_{i j}^{P}(G)=\beta_{i j}^{P / Q}(\bar{G})$.

- $H S_{G}(t)=\frac{H S_{\bar{G}}(t)}{(1-t)^{n-2}}$.

In particular $H S_{\bar{G}}(t)=h$ and $\nu(J)=\nu(\bar{J})$. Hence from now on we assume that $J \subseteq$ $P=k[x, y]$ and $G=P / J$ is Artinian with Hilbert function determined by $h$.

Let $L$ be the lex-segment ideal associated to $h$. Then $\nu(L)=d+1$. Let us denote by $p_{j}=\triangle h(j)$ and $q_{j}=\triangle^{2} h(j)$. Since the lex-segment ideal $L$ is Borel fixed, by EliahouKervaire's resolution (see [5]), the graded minimal free resolution of $L$ is the following

$$
0 \longrightarrow \bigoplus_{j=1}^{t} P^{\left|p_{c_{j}}\right|}\left(-c_{j}-1\right) \longrightarrow P(-d) \bigoplus_{j=1}^{t} P^{\left|p_{c_{j}}\right|}\left(-c_{j}\right) \longrightarrow L \longrightarrow 0,
$$

where $d \leq c_{1}<c_{2}<\cdots<c_{t}, \beta_{0}(L)=\sum_{j=1}^{t}\left|p_{c_{j}}\right|=d+1$ and $\left|p_{c_{j}}\right|$ denotes the number of generators of $L$ of degree $c_{j}$. By [13, Theorem 1.1] the minimal free resolution of $J$ is obtained from the minimal free resolution of $L$ by a sequence of consecutive zero cancellations. Therefore $\nu(J)=d+1-r$, where $r$ denotes the number of zero cancellations. Hence it is enough to compute the maximum number of zero cancellations that we may perform.

In the minimal free resolution of $L, P(-d)$ and $P\left(-c_{1}\right)$ cannot be cancelled since $c_{i}+1>$ $c_{1} \geq d$ for every $i=1, \ldots, t$. Now we have to compute $\beta_{0 c_{j}}(J)$ for $j \geq 2$. The zero cancellation at $c_{j}$-position with multiplicity $\alpha$ will be denoted by $\left(P^{\alpha}\left(-c_{j}\right), P^{\alpha}\left(-c_{j}\right)\right)$. A resolution admits a zero cancellation at $c_{j}$-th position only if $c_{j}=c_{j-1}+1$. Let us assume that we have the zero cancellation $\left(P^{\left|p_{c_{j-1}}\right|}\left(-c_{j}\right), P^{\left|p_{c_{j}}\right|}\left(-c_{j}\right)\right)$ at the $c_{j}$-th position. If $q_{c_{j}}=p_{c_{j}}-p_{c_{j-1}}<0$ then, after the above cancellation, we obtain $\left(0, P^{\left|q_{c_{j}}\right|}\left(-c_{j}\right)\right)$ and hence $\beta_{0 c_{j}}(J)=\left|q_{c_{j}}\right|$ and $\beta_{1 c_{j}}(J)=0$. If $q_{c_{j}}>0$ then the zero cancellation at $c_{j}$-th place can be replaced by $\left(P^{\left|q_{c_{j}}\right|}\left(-c_{j}\right), 0\right)$ having $\beta_{0 c_{j}}(J)=0$ and $\beta_{1 c_{j}}(J)=\left|q_{c_{j}}\right|$. If $q_{c_{j}}=0$ then it will be replaced by $(0,0)$ and in this case $\beta_{0 c_{j}}(J)=0$ and $\beta_{1 c_{j}}(J)=0$. In any case if we perform the zero cancellation at the $c_{j}$-th place then $\triangle^{2} h$ determines $\beta_{0 c_{j}}(J)$ and $\beta_{1 c_{j}}(J)$. Assume now $c_{j-1}+2 \leq c_{j} \leq c_{j+1}-2$, since we cannot perform zero cancellation at $c_{j}$ and $c_{j+1}$-th positions we have $p_{c_{j}-1}=0$ and $p_{c_{j}+1}=0$ and hence $\beta_{0 c_{j}}(J)=\left|p_{c_{j}}\right|=\left|p_{c_{j}}-p_{c_{j}-1}\right|=\left|q_{c_{j}}\right|$. In particular $q_{c_{j}}<0$. Moreover, $\beta_{1 c_{j}+1}(J)=$ $\left|p_{c_{j}}\right|=\left|p_{c_{j}+1}-p_{c_{j}}\right|=\left|q_{c_{j}+1}\right|$. In particular $q_{c_{j}+1}>0$. Thus if we perform all the 
possible consecutive zero cancellations in the minimal free resolution of $L$ then $\triangle^{2} h$ determine the graded Betti numbers of the minimal free resolution of $J$. Therefore we have $\nu(J)=\sum_{j \geq 1} \beta_{0 j}(J) \geq \sum_{i \in \mathcal{I}}\left|\triangle^{2} h(i)\right|$. Now if equality holds or equivalently if we perform all the possible consecutive zero cancellations in the minimal free resolution of $L$ then the minimal free resolution of $J$ has the following form

$$
0 \longrightarrow \bigoplus_{i \in \mathcal{J}} P^{\triangle^{2} h(i)}(-i) \longrightarrow \bigoplus_{i \in \mathcal{I}} P^{\left|\triangle^{2} h(i)\right|}(-i) \longrightarrow J \longrightarrow 0
$$

which implies that the graded Betti numbers of $J$ are uniquely determined by $h$ as follows

$$
\begin{aligned}
& \beta_{0 j}(J)= \begin{cases}\left|\triangle^{2} h(j)\right| & \text { for } j \in \mathcal{I} \\
0 & \text { otherwise }\end{cases} \\
& \beta_{1 j}(J)= \begin{cases}\left|\triangle^{2} h(j)\right| & \text { for } j \in \mathcal{J} \\
0 & \text { otherwise. }\end{cases}
\end{aligned}
$$

We apply the above result in the following situation of our major interest.

Corollary 1.2. Let $h:=\left(1,2, \ldots, d, h_{d}, \ldots, h_{s}\right)$ be an $O$-sequence and define the sets $\mathcal{I}:=\left\{j \mid \triangle^{2} h(j) \leq-1\right\}$ and $\mathcal{J}:=\left\{j \mid \triangle^{2} h(j) \geq 1\right\}$. Let $I \subseteq \mathfrak{n}^{2}$ an ideal of regular local ring $(S, \mathfrak{n})$ such that $h$ is the h-vector of $R=S / I$. Assume $G=g r_{\mathfrak{m}}(R)$ is Cohen-Macaulay, then

$$
\nu\left(I^{*}\right) \geq \sum_{i \in \mathcal{I}}\left|\triangle^{2} h(i)\right|
$$

If the equality holds, then

$$
\begin{aligned}
& \beta_{0 j}\left(I^{*}\right)= \begin{cases}\left|\triangle^{2} h(j)\right| & \text { for } j \in \mathcal{I} \\
0 & \text { otherwise }\end{cases} \\
& \beta_{1 j}\left(I^{*}\right)= \begin{cases}\left|\triangle^{2} h(j)\right| & \text { for } j \in \mathcal{J} \\
0 & \text { otherwise. }\end{cases}
\end{aligned}
$$

Remark 1.3. By the effective method proved by Bertella in [1], it is always possible to find an ideal attaining the minimal value according to Corollary 1.2. Let $h:=\left(1,2, \ldots, d, h_{d}, \ldots, h_{s}\right)$ be an $O$-sequence.

Define $\mathcal{I}:=\left\{j \mid \triangle^{2} h(j) \leq-1\right\}$ and $p:=\max \{|\triangle h(i)| \mid i=1, \ldots, s\}$. Then there exists an ideal $I$ of $S=k[[x, y]]$ such that

(1) $h=H F_{S / I}$,

(2) $\nu\left(I^{*}\right)=\sum_{i \in \mathcal{I}}\left|\triangle^{2} h(i)\right|$, and

(3) $\nu(I)=m$ for every integer $m$ such that $p+1 \leq m \leq \nu\left(I^{*}\right)$.

The following example will be useful to clarify the above remark. 
Example 1.4. Consider the following $O$-sequence:

$$
h=(1,2,3,4,5,6,7,8,9,10,10,10,9,8,8,5,3,3,2,0,0) .
$$

Then $\triangle h=(1,1,1,1,1,1,1,1,1,1,0,0,-1,-1,0,-3,-2,0,-1,-2,0)$ and $\triangle^{2} h=$ $=(1,0,0,0,0,0,0,0,0,0,0,-1,0,-1,0,1,-3,1,2,-1,-1,2)$.

Our goal is to construct an ideal $I \subseteq S=k[[x, y]]$ such that $h=H F_{S / I}$ and $\nu\left(I^{*}\right)=$ $\sum_{i \in \mathcal{I}}\left|\triangle^{2} h(i)\right|$. Then the order of $I$ is $d=10$ and the maximal jump in the Hilbert function is $p=\max _{i \geq 1}\{|\triangle h(i)|\}=3$. Moreover, by Equations (3) and (4), we have $4 \leq \nu(I) \leq 11$ and, by Theorem [1.1, we deduce $7 \leq \nu\left(I^{*}\right) \leq 11$. Bertella's construction suggests to realize $I^{*}$ by a suitable deformation of the Hilbert-Burch matrix associated to the (unique) lex-segment ideal $L$ such that $h=H F_{S / L}$. On his turn, the ideal $I$ will be obtained in the analogous way by the matrix associated to $I^{*}$.

It is easy to verify that

$$
L=\left(x^{10}, x^{9} y^{3}, x^{8} y^{5}, x^{7} y^{8}, x^{6} y^{9}, x^{5} y^{10}, x^{4} y^{12}, x^{3} y^{13}, x^{2} y^{16}, x y^{18}, y^{19}\right)
$$

is the lex-segment ideal associated to $h$. The minimal free resolution of $L$ is as follows:

$$
\begin{gathered}
0 \longrightarrow P(-13) \oplus P(-14) \oplus P^{3}(-16) \oplus P^{2}(-17) \oplus P(-19) \oplus P^{2}(-20) \longrightarrow \\
P(-10) \oplus P(-12) \oplus P(-13) \oplus P^{3}(-15) \oplus P^{2}(-16) \oplus P(-18) \oplus P^{2}(-19) \longrightarrow L \longrightarrow 0
\end{gathered}
$$

The above resolution admits at most 4 consecutive zero cancellations: $(P(-13), P(-13))$, $\left(P^{2}(-16), P^{2}(-16)\right)$ and $(P(-19), P(-19))$. Hence, by Theorem 1.1, $\nu\left(I^{*}\right) \geq 7$. For having $\nu\left(I^{*}\right)=7$ we have to perform all the above cancellations. Then we obtain the minimal free resolution of $I^{*}$ as follows

$$
\begin{gathered}
0 \rightarrow P(-14) \oplus P(-16) \oplus P^{2}(-17) \oplus P^{2}(-20) \longrightarrow \\
P(-10) \oplus P(-12) \oplus P^{3}(-15) \oplus P(-18) \oplus P(-19) \rightarrow I^{*} \longrightarrow 0
\end{gathered}
$$

with $\beta_{0 j}\left(I^{*}\right)=\left|\triangle^{2} h(j)\right|$ for $j \in \mathcal{I}$ and $\beta_{1 j}\left(I^{*}\right)=\left|\triangle^{2} h(j)\right|$ for $j \in \mathcal{J}$. Consider the Hilbert-Burch matrix associated to $L$, we may realize an ideal $I^{*}$ having the above resolution replaicing 0 by 1 in the entries corresponding to the zero cancellations. Then 
$I^{*}$ is the ideal generated by the maximal minors of the following matrix

$$
M:=\left(\begin{array}{cccccccccc}
y^{3} & 0 & 0 & 0 & 0 & 0 & 0 & 0 & 0 & 0 \\
-x & y^{2} & 0 & 0 & 0 & 0 & 0 & 0 & 0 & 0 \\
1 & -x & y^{3} & 0 & 0 & 0 & 0 & 0 & 0 & 0 \\
0 & 0 & -x & y & 0 & 0 & 0 & 0 & 0 & 0 \\
0 & 0 & 0 & -x & y & 0 & 0 & 0 & 0 & 0 \\
0 & 0 & 0 & 0 & -x & y^{2} & 0 & 0 & 0 & 0 \\
0 & 0 & 1 & 0 & 0 & -x & y & 0 & 0 & 0 \\
0 & 0 & 0 & 1 & 0 & 0 & -x & y^{3} & 0 & 0 \\
0 & 0 & 0 & 0 & 0 & 0 & 0 & -x & y^{2} & 0 \\
0 & 0 & 0 & 0 & 0 & 0 & 0 & 1 & -x & y \\
0 & 0 & 0 & 0 & 0 & 0 & 0 & 0 & 0 & -x
\end{array}\right)
$$

In particular $I^{*}=\left(x^{10}-2 x^{8} y^{2}-x^{6} y^{4}+4 x^{4} y^{6}-2 x^{2} y^{8},-x^{9} y^{3}+x^{7} y^{5}+2 x^{5} y^{7}-2 x^{3} y^{9},-x^{7} y^{8}+\right.$ $\left.x^{5} y^{10}+x^{3} y^{12}-x y^{14}, x^{6} y^{9}-x^{4} y^{11},-x^{5} y^{10}+x^{3} y^{12}, x^{2} y^{16}, y^{19}\right)$.

The resolution of $I^{*}$ admits the following three negative cancellations $(P(-14), P(-15))$, $(P(-16), P(-18))$ and $(P(-17), P(-19))$. Hence $4 \leq m=\nu(I) \leq 7$. For having an ideal with $\nu(I)=m$, by Rossi and Sharifan's result in [14, Remark 4.7] we have to perform $7-m$ negative cancellations. As before, by replacing in the matrix $M$ the entries 0's by 1 's in the positions corresponding to the negative cancellations, we obtain the wanted ideal. For instance, an ideal 4-generated whose leading ideal is $I^{*}$ :

$I=\left(x^{10}-2 x^{8} y^{2}-x^{6} y^{4}+4 x^{4} y^{6}-2 x^{2} y^{8}+x^{4} y^{9}+x^{3} y^{10}-x^{6} y^{6}-x^{5} y^{7}+x^{4} y^{8}+x^{3} y^{9}-x^{8} y^{3}+\right.$ $x^{6} y^{5}+x^{4} y^{7}-x^{2} y^{9},-x^{9} y^{3}+x^{7} y^{5}+2 x^{5} y^{7}-2 x^{3} y^{9}-x^{3} y^{12}-x^{2} y^{13}+x^{5} y^{9}+x^{4} y^{10}+x^{7} y^{6}-$ $\left.x^{5} y^{8}-x^{3} y^{10}+x y^{12}, x^{6} y^{9}-x^{4} y^{11}+y^{17}-x^{5} y^{10}+x^{3} y^{12},-x^{2} y^{15}-x y^{16},-x^{5} y^{10}+x^{3} y^{12}+y^{17}\right)$.

\section{When $I$ IS A COMPLETE INTERSECTION}

In this section we investigate the structure of $I^{*}$ when $\nu(I)=2$, that is, $I$ is a complete intersection in a regular local ring $S$ of dimension two. The results can be extended to higher dimension, provided $G$ is Cohen-Macaulay.

We prove that the numerical invariants of the graded minimal free resolution of $I^{*}$ are uniquely determined by the Hilbert function. This is quite unexpected because, in general, different Betti tables correspond to the same Hilbert function.

We recall that if $h$ is an $O$-sequence, then there exists a complete intersection ideal $I=(f, g) \subseteq S=k[[x, y]]$ such that $H F_{S / I}=h$ if and only if $|\triangle h(i)| \leq 1$ for every $i$.

Theorem 2.1. Let $h:=\left(1,2, \ldots, d, h_{d}, \ldots, h_{s}\right)$ be an $O$-sequence and define the sets $\mathcal{I}:=\left\{j \mid \triangle^{2} h(j) \leq-1\right\}$ and $\mathcal{J}:=\left\{j \mid \triangle^{2} h(j) \geq 1\right\}$. Then for all ideals I generated by $a$ regular sequence in a 2-dimensional regular local ring $S$ such that $h=H F_{S / I}$, the graded 
Betti numbers of $I^{*}$ are uniquely determined by $h$ as follows:

$$
\begin{aligned}
& \beta_{0 j}\left(I^{*}\right)= \begin{cases}\left|\triangle^{2} h(j)\right| & \text { for } j \in \mathcal{I} \\
0 & \text { otherwise, }\end{cases} \\
& \beta_{1 j}\left(I^{*}\right)= \begin{cases}\left|\triangle^{2} h(j)\right| & \text { for } j \in \mathcal{J} \\
0 & \text { otherwise. }\end{cases}
\end{aligned}
$$

Proof. Since $\nu(I)=2$, then by [1, Theorem 2.3] we have $p=\max _{i \geq 1}\{|\triangle h(i)|\}=1$. Let $L$ be the lex-segment ideal corresponding to $h$. By [14, Theorem 4.1] the Betti numbers of $I$ come from the Betti numbers of $L$ by a sequence of consecutive zero and negative cancellations. One can prove easily that when $I$ is a complete intersection, then there exists a unique diagram of cancellations which forces the Betti numbers of $I^{*}$. Then by Eliahou-Kervaire's resolution in [5] the minimal free resolution of $L$ is of the form

$$
0 \longrightarrow \bigoplus_{j=1}^{d} P\left(-c_{j}-1\right) \longrightarrow P(-d) \bigoplus_{j=1}^{d} P\left(-c_{j}\right) \longrightarrow L \longrightarrow 0
$$

where $d \leq c_{1}<c_{2}<\cdots<c_{d}$. Note that $P(-d)$ and $P\left(-c_{1}\right)$ can not be cancelled as $d \leq c_{1}<c_{i}+1$ for $i=1, \ldots, d$. Hence for having $\nu(I)=2$, all the remaining part $\bigoplus_{j \geq 2}^{d} P\left(-c_{j}\right)$ must be cancelled by zero or negative cancellations. Now $P\left(-c_{2}\right)$ can be only cancelled with $P\left(-c_{1}-1\right)$ (zero or negative cancellation). Going on inductively on $i$, we observe that for each $c_{i}$, we have only one candidate: $\left(P\left(-c_{i-1}-1\right), P\left(-c_{i}\right)\right)$ and the cancellation must be performed for reaching $\nu(I)=2$. In particular we have to perform all the zero cancellations in the minimal free resolution of $L$. Therefore $\nu\left(I^{*}\right)=\sum_{i \in \mathcal{I}}\left|\triangle^{2} h(i)\right|$ and then by Theorem 1.1(2) the Betti numbers of $I^{*}$ are uniquely determined by $h$ as

$$
\begin{aligned}
& \beta_{0 j}\left(I^{*}\right)= \begin{cases}\left|\triangle^{2} h(j)\right| & \text { for } j \in \mathcal{I} \\
0 & \text { otherwise }\end{cases} \\
& \beta_{1 j}\left(I^{*}\right)= \begin{cases}\left|\triangle^{2} h(j)\right| & \text { for } j \in \mathcal{J} \\
0 & \text { otherwise. }\end{cases}
\end{aligned}
$$

On the spirit of the results of Goto-Heinzer-Kim proved in [9], we present a deeper investigation of the structure of the leading ideal of a complete intersection by using the techniques of the present paper.

Let $(S, \mathfrak{n})$ be a 2-dimensional regular local ring and $I=(f, g)$ be a complete intersection ideal with $\mathfrak{n}$-adic valuations $v_{\mathfrak{n}}(f)=a \leq v_{\mathfrak{n}}(g)=b$ and $f^{*} \nmid g^{*}$. In [9] Goto, Heinzer and Kim have proved that if $\nu\left(I^{*}\right)=n$, then $I^{*}$ contains a homogeneous system of generators $\left\{\zeta_{i}\right\}$ such that $f^{*}=\zeta_{1}, g^{*}=\zeta_{2}$ and $\operatorname{deg} \zeta_{i}+2 \leq \operatorname{deg} \zeta_{i+1}$ for $2 \leq i \leq n-1$ and they 
described the Hilbert series of $G$ in terms of $c_{i}=$ the degree of $\zeta_{i}$ and the integers $d_{i}=$ the degrees of $G C D\left(\zeta_{1}, \ldots, \zeta_{i}\right)$.

Given a complete intersection ideal $I=(f, g)$, they associate a positive integer $n$ with $2 \leq n \leq a+1$, an ascending sequence of positive integers $\left(c_{1}=a, \ldots, c_{n}\right)$ and a descending sequence of integers $\left(d_{1}=c_{1}, d_{2}, \ldots, d_{n}=0\right)$ such that $c_{i+1}-c_{i}>d_{i-1}-d_{i}>0$ for each $i$ with $2 \leq i \leq n-1$. In [10, Theorem 2.3] Goto, Heinzer and Kim proved that these two sequence of integers $c_{i}$ 's and $d_{i}$ 's give also sufficient conditions for the existence of a complete intersection ideal $I$ whose leading ideal has these invariants.

In the following theorem we prove the analogous of [9, Theorem 1.2, 1.3], but we replace the sequence of $d_{i}$ 's with a sequence, say $e_{i}$ 's, directly related to the minimal free resolution of $I^{*}$. Obviously explicit relations among these integers exist and they will be discussed in Remark 2.3. The new approach allows us easier proofs because it takes advantage of the homological properties of the perfect ideal $I^{*}$.

We recall that important numerical information on a perfect homogeneous ideal of codimension two (in this case $I^{*}$ ) come from the Hilbert-Burch theorem: all the possible Hilbert functions (more in general the graded Betti numbers) of an ideal minimally generated by $n$ forms with assigned degrees $c_{1}, \ldots, c_{n}$ are in one to one correspondence with the $(n-1)$-tuples $\left(e_{2}, \ldots, e_{n}\right)$ such that $c_{i}<e_{i}<c_{i+1}, c_{1}+1 \geq n$ and $\sum_{i} c_{i}=\sum_{i} e_{i}$. In our case more conditions will be necessary because $I^{*}$ is the leading ideal of a complete intersection of given valuations. The theory of the cancellations come to our help. For our assumption we need a stronger condition given by item (3) in the following result.

Theorem 2.2. Let $(S, \mathfrak{n})$ be a regular local ring of dimension 2 and let $I=(f, g)$ be a complete intersection ideal in $S$ with $v_{\mathfrak{n}}(f)=d \leq v_{\mathfrak{n}}(g)=b$. Let $\nu\left(I^{*}\right)=n$. Then there exist two sequences of integers $\left(c_{1}, \ldots, c_{n}\right)$ and $\left(0=e_{1}, e_{2} \ldots, e_{n}\right)$ such that the following assertions hold true.

(1) $c_{1}=d$ and $c_{2}=b$,

(2) $c_{i}+2 \leq c_{i+1}$ for $i=2, \ldots, n-1$,

(3) $c_{i}+1 \leq e_{i}<c_{i+1}$ for $i=2, \ldots, n-1$ and $c_{n}+1 \leq e_{n}$,

(4) $\sum_{i=2}^{n}\left(e_{i}-c_{i}\right)=d$,

(5) $H S_{G}(t)=\frac{\sum_{i=1}^{n}\left(t^{e_{i}}-t^{c_{i}}\right)}{(1-t)^{2}}$,

(6) $e(G)=\frac{\sum_{i=1}^{n}\left[e_{i}\left(e_{i}-1\right)-c_{i}\left(c_{i}-1\right)\right]}{2}$, where $e(G)$ denotes the multiplicity of $G$,

(7) $a(G)=e_{n}-2$, where $a(G)$ denotes the a-invariant of $G$.

Proof. Let $H F_{S / I}$ be the Hilbert function of $S / I$ and let $L$ be the lex-segment ideal corresponding to $H F_{S / I}$. The minimal free resolution of $L$ by [5] has the following shape: 


$$
0 \longrightarrow \oplus_{j=1}^{d} P\left(-a_{j}-1\right) \longrightarrow P\left(-a_{0}\right) \oplus_{j=1}^{d} P\left(-a_{j}\right) \longrightarrow L \longrightarrow 0
$$

where $a_{0}=d=v_{\mathfrak{n}}(f)$ and $a_{1}=b=v_{\mathfrak{n}}(g)$. Note that in the resolution of $L, P\left(-a_{0}\right)$ and $P\left(-a_{1}\right)$ can not be cancelled since $a_{0} \leq a_{1}<a_{j}+1$ for $j=1, \ldots, d$. We consider the two sets of integers corresponding to the shifts in homological positions 0 and 1 in a minimal free resolution of $I^{*}$ :

$$
0 \longrightarrow \oplus_{j=2}^{n} P\left(-e_{j}\right) \longrightarrow P\left(-c_{1}\right) \oplus_{j=2}^{n} P\left(-c_{j}\right) \longrightarrow I^{*} \longrightarrow 0 .
$$

Then $c_{1}=a_{0}=d$ and $c_{2}=a_{1}=b$. Since the resolution of $I^{*}$ is obtained after performing all the possible zero cancellations we have $c_{i}+2 \leq c_{i+1}$ for $i=2, \ldots, n-1$. Since $\nu(I)=2$, by Rossi and Sharafan's result in [14, Remark 4.7] we have to perform $n-2$ negative cancellations. Hence we have $c_{i}+1 \leq e_{i}<c_{i+1}$ for $i=2, \ldots, n-1$. Condition (4) is well known and it follows by the projective dimension two.

From the resolution of $I^{*}$ we can compute the Hilbert series and by [3. Lemma 4.1.13], we have $H S_{G}(t)=\frac{\sum_{i=1}^{n}\left(t^{e_{i}}-t^{c_{i}}\right)}{(1-t)^{2}}$ and hence $e(G)=\frac{\sum_{i=1}^{n}\left[e_{i}\left(e_{i}-1\right)-c_{i}\left(c_{i}-1\right)\right]}{2}$. Since $\operatorname{dim} G=0$ the $a$-invariant of $G$ is $a(G)=\max \left\{t \mid G_{t} \neq 0\right\}=\max \left\{t \mid h_{t} \neq 0\right\}$. From the minimal free resolution of $L$ it is clear that $a(G)=a_{d}-1$. In the minimal free resolution of $L, P\left(-a_{d}-1\right)$ can never be cancelled since $a_{j}<a_{d}+1$ for $j=0,1, \ldots, d$ so it will remain in the minimal free resolution of $I^{*}$ and therefore $e_{n}=a_{d}+1$. Hence we have $a(G)=e_{n}-2$.

In Theorem 2.2 we observe that item (2) follows from item (3), nevertheless we think that it is interesting to outline that the degrees of the initial forms cannot be consecutive, except those of the first generators.

Remark 2.3. Goto-Heinzer-Kim in [9, Theorem 1.3] have expressed the Hilbert series of $G$ in terms of the sequence $c_{i}$ 's and $d_{i}$ 's as follows:

$$
H S_{G}(t)=\frac{\sum_{i=2}^{n} t^{d_{i}}\left(1-t^{d_{i-1}-d_{i}}\right)\left(1-t^{c_{i}-d_{i}}\right)}{(1-t)^{2}}
$$

where $d_{1}=c_{1}>d_{2}>\cdots>d_{n-1}>d_{n}=0$.

Now comparing this expression with Theorem 2.2 (5), we obtain for $i=2, \ldots n$

$$
e_{i}=c_{i}+\left(d_{i-1}-d_{i}\right)
$$

Let notation be as in Theorem 2.2. In the next theorem we prove that the two sequences of integers $c_{i}$ 's and $e_{i}$ 's are also sufficient for the existence of a complete intersection ideal $I$ whose leading ideal has these invariants. The result is the analogous of $[10$, Theorem $2.3]$. 
Theorem 2.4. Let $a$ and $b$ be positive integers with $a \leq b$ and consider the following data:

(1) An integer $n$ with $2 \leq n \leq a+1$.

(2) A sequence of integers $\left(a=c_{1}, b=c_{2}, c_{3}, \ldots, c_{n}\right)$ such that $c_{i}+2 \leq c_{i+1}$ for $i=2, \ldots, n-1$.

(3) A sequence of integers $\left(0=e_{1}, e_{2}, \ldots, e_{n}\right)$ such that $c_{i}+1 \leq e_{i}<c_{i+1}$ for $i=2, \ldots, n-1$ and $c_{n}+1 \leq e_{n}$ and $\sum_{i=2}^{n}\left(e_{i}-c_{i}\right)=a$.

For each system satisfying these conditions there exists an ideal $I=(f, g) \subseteq S=k[|x, y|]$ generated by a complete intersection with $v_{\mathfrak{n}}(f)=a$ and $v_{\mathfrak{n}}(g)=b$ such that $\nu\left(I^{*}\right)=n$ and these two sequences of integers $c_{i}$ 's and $e_{i}$ 's completely determine the minimal free resolution of $I^{*}$ as follows:

$$
0 \longrightarrow \oplus_{j=2}^{n} P\left(-e_{j}\right) \longrightarrow P\left(-c_{1}\right) \oplus_{j=2}^{n} P\left(-c_{j}\right) \longrightarrow I^{*} \longrightarrow 0 .
$$

Proof. From the two sequence of integers $c_{i}$ 's and $e_{i}$ 's let us define a numerical function as follows:

$$
q(j)=\left\{\begin{aligned}
-1 & \text { if } j=c_{i} \text { for } 3 \leq i \leq n \\
-2 & \text { if } j=c_{1}=c_{2} \\
-1 & \text { if } j=c_{1}, c_{2} \text { and } c_{1} \neq c_{2} \\
1 & \text { if } j=e_{i} \text { for } i=2, \ldots, n \\
0 & \text { otherwise. }
\end{aligned}\right.
$$

Then define another numerical function $p$ inductively as follows

$$
p(0)=1 \text { and } p(j)=p(j-1)+q(j) \text { for } j \geq 1 .
$$

Then we get

$$
p(j)=\left\{\begin{array}{rr}
1 & \text { if } 1 \leq j<c_{1} \\
-1 & \text { if } c_{i} \leq j<e_{i} \text { for } 2 \leq i \leq n \\
-1 & \text { if } j=c_{1}=c_{2} \\
0 & \text { otherwise }
\end{array}\right.
$$

Now define the numerical function $h$ inductively as follows

$$
h(0)=1 \text { and } h(j)=h(j-1)+p(j) \text { for } j \geq 1 .
$$

Then we can observe that

(1) $h(j)=j+1$ for $0 \leq j<c_{1}$

(2) $h(j) \geq h(j+1)$ for $j \geq c_{1}$

(3) $|h(j)-h(j-1)|=|p(j)| \leq 1$ for $j \geq 1$. 
Since $p(j)=-1$ for $c_{i} \leq j<e_{i}$ with $i=2, \ldots, n$ and $\sum_{i=2}^{n}\left(e_{i}-c_{i}\right)=c_{1}$ we have $h\left(e_{n}-2\right)=1 \geq h\left(e_{n}-1\right)=0=h(j)$ for all $j \geq e_{n}$. The above conditions imply that $h$ is an numerical function admissible for an Artinian graded $k$-algebra of codimension 2 . Note that $p$ and $q$ actually denote the $\triangle h$ and $\triangle^{2} h$ respectively. Let $\mathcal{I}:=\left\{j \mid \triangle^{2} h(j) \leq-1\right\}$ and $\mathcal{J}:=\left\{j \mid \triangle^{2} h(j) \geq 1\right\}$. By [1, Theorem 2.4] there exists a complete intersection ideal $I=(f, g)$ such that $H F_{S / I}=h$. Then by Theorem 2.1 the graded Betti numbers of $I^{*}$ are uniquely determined by $h$ as follows

$$
\begin{aligned}
& \beta_{0 j}\left(I^{*}\right)= \begin{cases}\left|\triangle^{2} h(j)\right| & \text { for } j \in \mathcal{I} \\
0 & \text { otherwise }\end{cases} \\
& \beta_{1 j}\left(I^{*}\right)= \begin{cases}\left|\triangle^{2} h(j)\right| & \text { for } j \in \mathcal{J} \\
0 & \text { otherwise. }\end{cases}
\end{aligned}
$$

and the minimal free resolution of $I^{*}$ can be expressed in terms of these two sequences of integers as follows:

$$
0 \longrightarrow \oplus_{j=2}^{n} P\left(-e_{j}\right) \longrightarrow P\left(-c_{1}\right) \oplus_{j=2}^{n} P\left(-c_{j}\right) \longrightarrow I^{*} \longrightarrow 0
$$

We give a concrete example for clarifying the above result.

Example 2.5. Consider the two sequences of integers $(4,5,8,11)$ and $(6,9,13)$ satisfying the conditions of Theorem 2.4. We will exhibit a complete intersection ideal $I=(f, g) \subseteq$ $k[[x, y]]$ such that the numerical invariants of the minimal free resolution of $I^{*}$ will be completely determined by these two sequence of integers, see equation (66). By Theorem 2.2(5) the Hilbert series corresponding to the two sequences $(4,5,8,11)$ and $(6,9,13)$ is given as

$$
\begin{aligned}
H S_{G}(t) & =\frac{\left(1-t^{4}+t^{6}-t^{5}+t^{9}-t^{8}+t^{13}-t^{11}\right)}{(1-t)^{2}} \\
& =1+2 t+3 t^{2}+4 t^{3}+4 t^{4}+3 t^{5}+3 t^{6}+3 t^{7}+2 t^{8}+2 t^{9}+2 t^{10}+t^{11}
\end{aligned}
$$

and the lex-segment ideal with the above Hilbert series is $L=\left(x^{4}, x^{3} y^{2}, x^{2} y^{6}, x y^{10}, y^{12}\right)$. The minimal free resolution of $L$ is of the form

$0 \longrightarrow P(-6) \oplus P(-9) \oplus P(-12) \oplus P(-13) \longrightarrow P(-4) \oplus P(-5) \oplus P(-8) \oplus P(-11) \oplus P(-12) \longrightarrow L$

The resolution of $L$ admits one zero cancellation $(P(-12), P(-12))$. The resolution of $I^{*}$ is obtained by performing the zero cancellation as follows

$$
0 \longrightarrow P(-6) \oplus P(-9) \oplus P(-13) \longrightarrow P(-4) \oplus P(-5) \oplus P(-8) \oplus P(-11) \longrightarrow I^{*}
$$


By Bertella's construction as in Example 1.4, then $I^{*}=\left(-x y^{10}, x^{2} y^{6}-y^{8},-x^{3} y^{2}+\right.$ $\left.x y^{4}, x^{4}-x^{2} y^{2}\right)$ is generated by the maximal minors of the following matrix

$$
\left(\begin{array}{cccc}
y^{2} & 0 & 0 & 0 \\
-x & y^{4} & 0 & 0 \\
0 & -x & y^{4} & 0 \\
0 & 0 & -x & y^{2} \\
0 & 0 & 1 & -x
\end{array}\right)
$$

The resolution of $I^{*}$ admits two negative cancellations : $(P(-6), P(-8))$ and $(P(-9), P(-11))$. By performing the negative cancellation we obtain the minimal free resolution of $I$, where $I=\left(x y^{6}-x^{3} y^{2}+x y^{4},-2 x^{2} y^{4}+y^{6}+x^{4}-x^{2} y^{2}\right)$ is generated by the maximal minors of the following matrix

$$
\left(\begin{array}{cccc}
y^{2} & 0 & 0 & 0 \\
-x & y^{4} & 0 & 0 \\
1 & -x & y^{4} & 0 \\
0 & 1 & -x & y^{2} \\
0 & 0 & 1 & -x
\end{array}\right)
$$

In [9, Theorem 1.6], Goto, Heinzer and Kim extended their results [9, Theorem 1.2 and 1.3] to dimension $s>2$, provided $G$ is Cohen-Macaulay. Using our approach the extension is immediate because the numerical invariants of the free resolution do not change modulo a regular sequence.

Theorem 2.6. Let $(S, \mathfrak{n})$ be a regular local ring of dimension $s>2$. Let $I=(f, g)$ be an ideal generated by a regular sequence in $S$ and let $R=S / I$ and $\mathfrak{m}=\mathfrak{n} / I$. We put $G:=$ $g r_{\mathfrak{m}}(R)$. If $G$ is Cohen-Macaulay ring and $\nu\left(I^{*}\right)=n$, then there exists two sequences of integers $\left(c_{1}, \ldots, c_{n}\right)$ and $\left(e_{1}=0, e_{2}, \ldots, e_{n}\right)$ satisfying the following conditions:

(1) $c_{1}=v_{\mathfrak{n}}(f)$ and $c_{2}=v_{\mathfrak{n}}(g)$

(2) $c_{i}+2 \leq c_{i+1}$ for $i=2, \ldots, n-1$.

(3) $c_{i}+1 \leq e_{i}<c_{i+1}$ for $i=2, \ldots, n-1$ and $c_{n}+1 \leq e_{n}$.

(4) $\sum_{i=2}^{n}\left(e_{i}-c_{i}\right)=v_{\mathfrak{n}}(f)$.

(5) $H S_{G}(t)=\frac{\sum_{i>1}\left(t^{e_{i}}-t^{c_{i}}\right)}{(1-t)^{s}}$.

(6) $a(G)=e_{n}-s$.

Proof. We may assume that the residue field $k$ is infinite. Let $\mathfrak{n}=\left(x_{1}, x_{2}, \ldots, x_{s}\right)$. Since $G$ is Cohen-Macaulay of dimension $s-2$, after a change of coordinates we may assume that $Q=\left(x_{3}^{*}, \ldots, x_{s}^{*}\right)$ is generated by a $G$-regular sequence. Let $\mathfrak{q}=\left(x_{3}, \ldots, x_{s}\right)$ and consider $\bar{S}=S / \mathfrak{q}$ with maximal ideal $\overline{\mathfrak{n}}=\mathfrak{n} / \mathfrak{q}$. Let $\bar{I}=(\bar{f}, \bar{g})$ where $\bar{f}, \bar{g}$ denote the images of $f$ and $g$ in $\bar{S}$, respectively. Then $\bar{I}$ is a parameter ideal in $\bar{S}$ and hence 
$\bar{R}=\bar{S} / \bar{I}$ is the Artinian local ring of embedding dimension two with maximal ideal $\overline{\mathfrak{m}}=\overline{\mathfrak{n}} / \bar{I}$. Since $G$ is Cohen-Macaulay we have $\operatorname{gr}_{\overline{\mathfrak{m}}}(\bar{R}) \simeq G / Q=\bar{G}$. We put $P:=g r_{\mathfrak{n}}(S)$. Now the following facts hold:

- $\beta_{i j}^{P}(G)=\beta_{i j}^{P / Q}(\bar{G})$.

- $H S_{G}(t)=\frac{H S_{\bar{G}}(t)}{(1-t)^{s-2}}$

$-a(G)=a(\bar{G})-(s-2)$

Thus by passing to $\bar{R}$ the result follows by Theorem 2.2 .

Remark 2.7. From Theorem 2.2, it follows that the Hilbert series of $G$ is uniquely determined by two sequences of integers $\left(c_{1}, c_{2}, \ldots, c_{n}\right)$ and $\left(e_{1}=0, e_{2}, \ldots, e_{n}\right)$. Usually the sequence $\left(c_{1}, \ldots, c_{n}\right)$ does not uniquely determine the Hilbert series of $G$. But if $\nu\left(I^{*}\right)=c_{1}+1$, then the minimal free resolution of $P / I^{*}$ is same as the minimal free resolution of the lex segment ideal associated and in that case we know that $e_{i}=c_{i}+1$ for $=2, \ldots, n$. Hence the Hilbert series of $G$ is uniquely determined by the sequence $\left(c_{1}, c_{2}, \ldots, c_{n}\right)$.

Next example shows that in general the sequence $\left(c_{1}, c_{2}, \ldots, c_{n}\right)$ does not determine uniquely the Hilbert series of $G$.

Example 2.8. Let $(S, \mathfrak{n})$ be a regular local ring and $I$ be a complete intersection ideal. Given the admissible sequence $\left(c_{1}, c_{2}, c_{3}, c_{4}\right)=(4,5,8,11)$, then, by Theorem 2.4 , the possible values for $\left(e_{2}, e_{3}, e_{4}\right)$ are

$$
(6,9,13),(6,10,12) \text { and }(7,9,12) \text {. }
$$

They correspond to three different Hilbert series of $G$ and each of them is realizable.

(1) The two sequence of integers $(4,5,8,11)$ and $(6,9,13)$ have already been discussed in Example 2.5.

(2) The Hilbert series corresponding to the two sequences $(4,5,8,11)$ and $(6,10,12)$ is given by

$$
\begin{aligned}
H S_{G}(t) & =\frac{\left(1-t^{4}+t^{6}-t^{5}+t^{10}-t^{8}+t^{12}-t^{11}\right)}{(1-t)^{2}} \\
& =1+2 t+3 t^{2}+4 t^{3}+4 t^{4}+3 t^{5}+3 t^{6}+3 t^{7}+2 t^{8}+t^{9}+t^{10}
\end{aligned}
$$

and the corresponding lex-segment ideal is $L=\left(x^{4}, x^{3} y^{2}, x^{2} y^{6}, x y^{8}, y^{11}\right)$. The minimal free resolution of $L$ is of the form

$0 \rightarrow P(-6) \oplus P(-9) \oplus P(-10) \oplus P(-12) \longrightarrow P(-4) \oplus P(-5) \oplus P(-8) \oplus P(-9) \oplus P(-11) \longrightarrow L \rightarrow 0$. 
The resolution of $L$ admits only one zero cancellation $(P(-9), P(-9))$. The resolution of $I^{*}$ is obtained by performing the zero cancellation as follows

$0 \longrightarrow P(-6) \oplus P(-10) \oplus P(-12) \longrightarrow P(-4) \oplus P(-5) \oplus P(-8) \oplus P(-11) \longrightarrow I^{*} \longrightarrow 0$.

For instance $I^{*}=\left(x^{4}-x^{2} y^{2},-x^{3} y^{2}+x y^{4}, x^{2} y^{6}, y^{11}\right)$ is generated by the maximal minors of the following matrix

$$
\left(\begin{array}{cccc}
y^{2} & 0 & 0 & 0 \\
-x & y^{4} & 0 & 0 \\
0 & -x & y^{2} & 0 \\
0 & 1 & -x & y^{3} \\
0 & 0 & 0 & -x
\end{array}\right)
$$

The resolution of $I^{*}$ admits two negative cancellations : $(P(-6), P(-8))$ and $(P(-10), P(-11))$. By performing the negative cancellations we obtain the minimal free resolution of $I$, where $I=\left(x y^{5}-x^{3} y^{2}+x y^{4}, y^{7}-x^{2} y^{4}-x^{2} y^{3}+x^{4}-x^{2} y^{2}\right)$ is generated by the maximal minors of the following matrix

$$
\left(\begin{array}{cccc}
y^{2} & 0 & 0 & 0 \\
-x & y^{4} & 0 & 0 \\
1 & -x & y^{2} & 0 \\
0 & 1 & -x & y^{3} \\
0 & 0 & 1 & -x
\end{array}\right)
$$

(3) The Hilbert series corresponding to the two sequences $(4,5,8,11)$ and $(7,9,12)$ is given as

$$
\begin{aligned}
H S_{G}(t) & =\frac{\left(1-t^{4}+t^{7}-t^{5}+t^{9}-t^{8}+t^{12}-t^{11}\right)}{(1-t)^{2}} \\
& =1+2 t+3 t^{2}+4 t^{3}+4 t^{4}+3 t^{5}+2 t^{6}+2 t^{7}+t^{8}+t^{9}+t^{10}
\end{aligned}
$$

and the lex segment ideal with the above Hilbert function is $L=\left(x^{4}, x^{3} y^{2}, x^{2} y^{4}, x y^{7}, y^{11}\right)$. By repeating the above procedure we obtain $I^{*}=\left(x^{4}-x^{2} y^{2},-x^{3} y^{2},-x y^{7}, y^{11}\right)$ and $I=\left(x y^{6}+x y^{5}-x^{3} y^{2},-x^{2} y^{4}+y^{6}-x^{2} y^{3}+x^{4}-x^{2} y^{2}\right)$.

\section{WHEN $\nu(I)=3$}

In this section we give a more precise upper bound on the number of generators of $I^{*}$, provided $\nu(I)=3$. It is clear that the method used in the above section suggests a procedure for getting information on the Betti table of $I^{*}$ anyway the number of 
generators of $I$ will be fixed. The problem is that the graph of the possible cancellations is too complicate for a general description. Let $h$ be an $O$-sequence and let us define

$$
\begin{gathered}
\mathcal{H}=\left\{i \mid \triangle^{2} h(i)=0, \triangle h(i)=-1\right\}, \\
\mathcal{I}=\left\{j \mid \triangle^{2} h(j) \leq-1\right\} \quad \text { and } \quad \mathcal{J}=\left\{j \mid \triangle^{2} h(j) \geq 1\right\} .
\end{gathered}
$$

Theorem 3.1. Let $h:=\left(1,2, \ldots, d, h_{d}, \ldots, h_{s}\right)$ be an $O$-sequence and let $I$ be an ideal of height two of a 2-dimensional regular local ring $S$ such that $H F_{S / I}=h$. If $\nu(I)=3$, then

$$
\sum_{\triangle^{2} h(i) \leq-1}\left|\triangle^{2} h(i)\right| \leq \nu\left(I^{*}\right) \leq \sum_{\triangle^{2} h(i) \leq-1}\left|\triangle^{2} h(i)\right|+\sum_{i \geq 0} \delta_{i}(\mathcal{H})
$$

where $\delta_{i}(\mathcal{H})= \begin{cases}1 & \text { if } i \in \mathcal{H} \\ 0 & \text { otherwise . }\end{cases}$

Proof. Notice that $I^{*}$ is a homogeneous perfect ideal of height two in $P:=g r_{\mathfrak{n}}(S)$. By Theorem 1.1, we have $\sum_{i \in \mathcal{I}}\left|\triangle^{2} h(i)\right| \leq \nu\left(I^{*}\right)$. Since $\nu(I)=3$ then by [1, Theorem 2.3] we have $p=\max _{i \geq 1}\{|\triangle h(i)|\} \leq 2$. Let $L$ be the lex-segment ideal corresponding to the numerical function $h$. Let us denote by $p_{j}:=\triangle h(j)$. Since $L$ is a Borel fixed ideal by [5] the minimal free resolution of $L$ is of the form

$$
0 \longrightarrow \bigoplus_{j=1}^{t} P^{\left|p_{c_{j}}\right|}\left(-c_{j}-1\right) \longrightarrow P(-d) \bigoplus_{j=1}^{t} P^{\left|p_{c_{j}}\right|}\left(-c_{j}\right) \longrightarrow L \longrightarrow 0
$$

where $d \leq c_{1}<c_{2}<\ldots<c_{t}$. By [14, Theorem 4.1] the Betti numbers of $I$ come from the Betti numbers of $L$ by performing a sequence of consecutive zero and negative cancellations and the minimal free resolution of $I$ is of the form

$$
0 \longrightarrow F_{2}=S^{2} \longrightarrow F_{1}=S^{3} \longrightarrow I \longrightarrow 0 .
$$

By [13, Theorem 1.1] the Betti numbers of $I^{*}$ come from the Betti numbers of $L$ by a sequence of consecutive zero cancellation. Thus in order to have the upper bound of the number of generators of $I^{*}$ we need to find how many zero cancellations in the minimal free resolution of $L$ can be replaced by negative cancellations. Let us assume that we have a zero cancellation at the $c_{j}$-th place. Then we have $c_{j-1}+1=c_{j}$. There are three possibilities for $\triangle^{2} h\left(c_{j}\right)$ namely $-1,1$ or 0 as $\left|p_{c_{j}}\right| \leq 2$ for all $j=1, \ldots t$. First assume that we have $\triangle^{2} h\left(c_{j}\right)=1$ then the resolution will be of the form $\longrightarrow \cdots P^{2}\left(-c_{j}\right) \oplus P\left(-c_{j}-1\right) \oplus \cdots \longrightarrow \cdots P^{2}\left(-c_{j-1}\right) \oplus P\left(-c_{j}\right) \oplus P^{\left|p_{c_{j+1}}\right|} P\left(-c_{j+1}\right) \cdots$ If we don't perform the zero cancellation $\left(P\left(-c_{j}\right), P\left(-c_{j}\right)\right)$ then $\sum_{i=j-1}^{t} \beta_{1 c_{i}+1}(L)=$ $\sum_{i=j+1}^{t} \beta_{0 c_{i}}(L)+3$ which forces $\operatorname{rank} F_{2}=3$ which is a contradiction. Thus the zero cancellation at the $c_{j}$-th place can not be replaced by negative cancellation. 
Next assume that we have a zero cancellation at the $c_{j}$-th place with $\triangle^{2} h\left(c_{j}\right)=-1$ then the resolution will be of the form

$$
\longrightarrow \cdots P\left(-c_{j}\right) \oplus P^{2}\left(-c_{j}-1\right) \oplus \cdots \longrightarrow \cdots P\left(-c_{j-1}\right) \oplus P^{2}\left(-c_{j}\right) \oplus P^{\left|p_{c_{j+1}}\right|} P\left(-c_{j+1}\right) \cdots
$$

If we don't perform the zero cancellation $\left(P\left(-c_{j}\right), P\left(-c_{j}\right)\right)$ then $\sum_{i=j-1}^{t} \beta_{1 c_{i}+1}(L)=$ $\sum_{i=j+1}^{t} \beta_{0 c_{i}}(L)+3$ which forces $\operatorname{rank} F_{2}=3$ which is a contradiction. Thus the zero cancellation at the $c_{j}$-th place can not be replaced by negative cancellation.

Let us assume that we have a zero cancellation at the $c_{j}$-th place and $\triangle^{2} h\left(c_{j}\right)=0$. It can happen in the following two ways. First consider the following case:

$\longrightarrow \cdots P^{2}\left(-c_{j}\right) \oplus P^{2}\left(-c_{j}-1\right) \oplus \cdots \longrightarrow \cdots P^{2}\left(-c_{j-1}\right) \oplus P^{2}\left(-c_{j}\right) \oplus P^{\left|p_{c_{j+1}}\right|} P\left(-c_{j+1}\right) \cdots$

where $\triangle h\left(c_{j}\right)=-2$. If we don't perform both the zero cancellation $\left(P^{2}\left(-c_{j}\right), P^{2}\left(-c_{j}\right)\right)$ then $\sum_{i=j-1}^{t} \beta_{1 c_{i}+1}(L)=\sum_{i=j+1}^{t} \beta_{0 c_{i}}(L)+4$ which forces $\operatorname{rank} F_{2}=4$ which is a contradiction. Thus the zero cancellation at the $c_{j}$-th place can not be replaced by negative cancellation. Next consider the following case

$$
\longrightarrow \cdots P\left(-c_{j}\right) \oplus P\left(-c_{j}-1\right) \oplus \cdots \longrightarrow \cdots P\left(-c_{j-1}\right) \oplus P\left(-c_{j}\right) \oplus P^{\left|p_{c_{j+1}}\right|} P\left(-c_{j+1}\right) \cdots
$$

where $\triangle h\left(c_{j}\right)=-1$ If we don't perform the zero cancellation $\left(P\left(-c_{j}\right), P\left(-c_{j}\right)\right)$ then $\sum_{i=j-1}^{t} \beta_{1 c_{i}+1}(L)=\sum_{i=j+1}^{t} \beta_{0 c_{i}}(L)+2$ which forces rank $F_{2}=2$. Thus the zero cancellation $\left(P\left(-c_{j}\right), P\left(-c_{j}\right)\right)$ can be replaced by a negative cancellation and it will increase the number of generators of $I^{*}$. Therefore we have

$$
\nu\left(I^{*}\right) \leq \sum_{\triangle^{2} h(i) \leq-1}\left|\triangle^{2} h(i)\right|+\sum_{i \geq 0} \delta_{i}(\mathcal{H})
$$

Goto-Heinzer-Kim in [8, Corollary 1.3] proved that if $I$ is a complete intersection ideal of codimension two, then $I^{*}$ is a perfect ideal provided $\nu\left(I^{*}\right)=3$. Inspired by this result we may ask if, given a perfect ideal $I$ of codimension two, the condition $\nu\left(I^{*}\right)=\nu(I)+1$ could be sufficient for having $I^{*}$ perfect. At the moment the answer is unknown. The authors thank Youngsu Kim for pointing out a mistake in a related example which appears in a preliminary version of this paper.

\section{REFERENCES}

[1] V. Bertella Hilbert function of local Artinian level rings in codimension 2, Journal of Algebra, 321 (2009), 1429-1442.

[2] J. Briancon, A. Iarrobino, Dimension of the punctual Hilbert Scheme, Journal of Algebra, 55 (1978), 536-544.

[3] W. Bruns and J. Herzog, Cohen-Macaulay Rings, Revised Edition, Cambridge University Press, 1998. 
[4] CoCoA Team, CoCoA: a system for doing Computations in Commutative Algebra, Avail- able at http://cocoa.dima.unige.it

[5] S. Eliahou and M. Kervaire, Minimal resolutions of some monomial ideals, Journal of Algebra 129 (1990), 1-25.

[6] J. Elias, A sharp bound for the minimal number of generators of perfect height two ideals, Manuscripta Math. 55 (1986), 93-99.

[7] J. Elias, L. Robbiano and G. Valla, Number of generators of ideals, Nagoya Math. J. 123 (1991), 39-76.

[8] S. Goto, W. Heinzer and M. Kim, The leading ideal of a complete intersection of height two, Journal of Algebra, 298 (2006), 238-247.

[9] S. Goto, W. Heinzer and M. Kim, The leading ideal of a complete intersection of height two, Part II, Journal of Algebra, 312 (2007), 709-732.

[10] S. Goto, W. Heinzer and M. Kim, The leading ideal of a complete intersection of height two in a 2-dimensional regular local ring, Communications in Algebra, 36 (2008), 1901-1910.

[11] A. Iarrobino, Punctual Hilbert schemes, Mem. Amer. Math. Soc. 188(1977).

[12] S.C. Kothari, The local Hilbert function of a pair of plane curves, Proc. Amer. Math. Soc., 72(3) (1978), 439-442.

[13] I. Peeva, Consecutive cancellations in betti numbers, Proc. Amer. Math. Soc., 132 (2004), 3503-3507.

[14] M. E. Rossi and L. Sharifan, Consecutive cancellations in Betti numbers of local rings, Proc. Amer. Math. Soc., 138(1) (2010), 61-73.

[15] J. D. Sally, Bounds for number of generators of Cohen-Macaulay ideals, Pac. J. Math. , 63 (1976), 517-520.

[16] J. D. Sally, Number of generators of ideals in local rings, Lecture Notes in Pure and Applied Mathematics, 35 New York, Marcel Dekker (1978).

Department of Mathematics, Indian Institute of Science Bangalore, 5600012, India

E-mail address: mousumi@math.iisc.ernet.in

Dipartimento Di Matematica, Universita’ Di Genova, Via Dodecaneso 35, 16146, Genova, ITALY

E-mail address: rossim@dima.unige.it 